\title{
Association of $O G G 1$ Ser326Cys polymorphism and pancreatic cancer susceptibility: evidence from a meta-analysis
}

\author{
Yulan Yan • Xu Chen • Taijie Li • Meng Li • \\ Hongjie Liang
}

Received: 19 June 2013 / Accepted: 14 October 2013 / Published online: 3 November 2013

(C) The Author(s) 2013. This article is published with open access at Springerlink.com

\begin{abstract}
The 8-oxoguanine DNA glycosylase (OGG1) gene has been considered to be associated with cancer susceptibility. The $O G G 1$ Ser326Cys polymorphism has been reported to be associated with pancreatic cancer (PC), but the published studies have yielded inconsistent results. For better understanding of the effect of $O G G 1$ Ser326Cys polymorphism on PC susceptibility, a meta-analysis was performed. All eligible studies were identified through a search of PubMed, Excerpta Medica Database (Embase), Elsevier Science Direct, and Chinese Biomedical Literature Database before May 2013. The association between the OGG1 Ser326Cys polymorphism and PC risk was conducted by odds ratios (ORs) and $95 \%$ confidence intervals (CIs). A total of five case-control studies with 1,690 cases and 3,650 controls were eventually collected. Overall, we found that OGG1 Ser326Cys polymorphism was not associated with PC susceptibility (Cys/Cys vs. Ser/Ser: OR $=0.95,95 \% \mathrm{CI}=$ $0.80-1.14$; Cys/Cys vs. Ser/Ser+Ser/Cys: $\mathrm{OR}=0.95,95 \%$ $\mathrm{CI}=0.78-1.14$; $\mathrm{Cys} / \mathrm{Cys}+\mathrm{Ser} / \mathrm{Cys}$ vs. Ser/Ser $(\mathrm{OR}=1.00$, $95 \% \mathrm{CI}=0.89-1.12)$ ). In the subgroup analysis based on
\end{abstract}

Yulan Yan and $\mathrm{Xu}$ Chen contributed equally to this work.

Y. Yan $\cdot$ X. Chen $\cdot$ T. Li $\cdot$ M. Li $\cdot$ H. Liang $(\bowtie)$

Department of Clinical Laboratory, First Affiliated Hospital of

Guangxi Medical University, 6 Shuangyong Road, Nanning,

Guangxi 530021, People's Republic of China

e-mail: xijunshi56234@163.com

Y. Yan

e-mail: xueying201120521@163.com

X. Chen

e-mail: chenxu16300@163.com

T. Li

e-mail: 715085562@qq.com

M. Li

e-mail: 1037106698@qq.com ethnicity, source of control, sample size, and genotyping method, no significant association was found in any genetic models. This meta-analysis suggests that the $O G G 1$ Ser326Cys polymorphism may not associated with PC susceptibility. Considering the limited sample size and ethnicity included in the meta-analysis, further larger scaled and well-designed studies are needed to confirm our results.

Keywords $O G G 1$ - Pancreatic cancer · Polymorphism · Meta-analysis

\section{Introduction}

Pancreatic cancer (PC) is one of the most fatal malignant tumors among human; although infrequent, it takes up $3 \%$ of all reported cases of cancer and it is the fourth most common cause of cancer-related deaths in the USA and the eighth worldwide [1-3]. What was worse, the prognosis of PC is very poor; the 5 -year survival rate is less than $5 \%$ even with the surgical and chemotherapy intervention. There were 44, 000 new cases diagnosed and 37,000 deaths from PC in 2012 [4-6]. The situation has not significantly changed over the past several decades [7]. It is extremely important for us to find more effective methods to treat PC. So, having a good knowledge of the molecular basis of PC is necessary [8,9]. A large number of studies have been carried out to explore potential risk factors of PC. Several factors have been considered as risk factors, such as diabetes [9], heavy alcohol consumption [10], smoking [11], and sucrose intake [12]. However, not all people exposed to those risk factors develop $\mathrm{PC}$, suggesting a genetic contribution to the development of $\mathrm{PC}[13,14]$.

The 8-oxoguanine DNA glycosylase (OGG1) gene, located on chromosome $3 \mathrm{p} 26$, is one of the component of 
BER pathway, plays a key role in repairing damaged DNA. It performs the initial step of recognizing the 8-hydroxyguanine damage which is highly mutagenic and a major form of oxidative DNA damage $[15,16]$. The $O G G 1$ gene has at least 20 validated sequence variants, and one of the most studied functional polymorphism is Ser326Cys (exon 7 of the $O G G 1$ gene, rs1052133), which with an acid substitution of serine (Ser) with cysteine (Cys) at codon 326, resulting from a $\mathrm{C}$ to $\mathrm{G}$ transversion at 1245 position, has been reported to affect the $O G G 1$ function and to be associated with cancer susceptibility [17-21]. It is reported that the 326Cys allele had reduced DNA repair activity and was related with cancer risk $[20,21]$. Up to now, many reports have evaluated the relationship between the OGG1 Ser326Cys polymorphism and PC risk. However, the results from these studies were inconsistent [22-26]. In order to assess the association between the $O G G 1$ Ser326Cys polymorphism and PC risk accurately, we carried out a meta-analysis.

\section{Materials and methods}

\section{Literature search}

We searched the relevant articles through the search engines such as PubMed, Excerpta Medica Database (Embase), Elsevier Science Direct, and Chinese Biomedical Literature Database using the search terms: "OGG1, OGH1, or OGG1", "variant or variation or polymorphism", and "pancreatic cancer" (last search was updated on 30 May 2013) without language restrict.

Inclusion and exclusion criteria

Studies were selected if they satisfied the following criteria: (1) estimating the association of $O G G 1$ Ser326Cys polymorphisms with PC risk, (2) case-control studies, (3) offering enough information for estimating the odds ratios (ORs) with the corresponding $95 \%$ confidence intervals (CIs). The exclusion criteria were: (1) not case-control studies that evaluated the association between $O G G 1$ polymorphism and PC risk; (2) case reports, letters, and reviews; and (3) controls were not consistent in HWE.

\section{Data extraction}

All the data were extracted by two investigators (YY and XC) independently and the result was reviewed by a third investigator (HL). From each study, the following information were collected: first author, year of publication, country and ethnicity of the study population, the number of cases and controls, genotyping methods and genotype distribution of cases and controls, and source of control.

Statistical analysis

To test whether the population of control conformed to Hardy-Weinberg equilibrium, a chi-square test was applied. The homogeneity among the studies was verified by Chi square-based $Q$ test [27] if there is a significant $Q$ statistic $(P<0.10)$ that indicated heterogeneity across studies, the pooled OR estimate of all studies would be calculated by the fixed-effects model (the Mantel-Haenszel method) [28]; otherwise, a random effects model (the DerSimonian and Laird method) would be used [29]. The strength of the association between $O G G 1$ Ser326Cys polymorphism and $\mathrm{PC}$ risk was measured by OR with $95 \% \mathrm{CI}$. The statistical significance of the summary OR was determined with the $Z$ test. The publication bias was tested by funnel plot and Egger's linear regression test [30, 31]. Sensitivity was analysis by sequential omission of individual study one a time or by omitting studies without high quality was performed to assess the stability of the results [32]. All statistical tests for this meta-analysis were performed with STATA version 9.0 (Stata Corporation, College Station, TX, USA).

\section{Results}

Studies characteristics

The general characteristics of eligible studies were listed in Table 1. After selecting based on the inclusion and exclusion criteria listed above, a total of five eligible studies with 1,690 cases and 3,650 controls were included in our meta-analysis [22-26]. The population of controls in each study was consistent with HWE $(P>0.05)$.
Table 1 Main characteristics of studies included in the meta-analysis

HWE Hardy-Weinberg equilibrium, $H B$ hospital based, $P B$ population based

\begin{tabular}{llllllll}
\hline First author & Year & Country & Ethnicity & $\begin{array}{l}\text { Genotyping } \\
\text { method }\end{array}$ & $\begin{array}{l}\text { Source of } \\
\text { control }\end{array}$ & Cases/controls & $\begin{array}{l}\text { HWE } \\
\text { control }\end{array}$ \\
\hline Nakao & 2012 & Japan & Asian & Taq Man & HB & $185 / 1,465$ & Yes \\
Li & 2009 & USA & Caucasians & Taq Man & HB & $722 / 773$ & Yes \\
Duell & 2008 & USA & Caucasians & Masscode & PB & $126 / 330$ & Yes \\
McWilliams & 2008 & USA & Caucasians & SNPstream & PB & $469 / 599$ & Yes \\
Zhang & 2011 & USA & Caucasians & Taq Man & PB & $188 / 483$ & Yes \\
\hline
\end{tabular}


Table 2 Meta-analysis of $O G G 1$ Ser326Cys polymorphism with the PC risk

\begin{tabular}{|c|c|c|c|c|c|c|c|c|c|}
\hline \multirow[t]{2}{*}{ Study group } & \multirow[t]{2}{*}{$N$} & \multicolumn{2}{|l|}{ Cys vs Ser } & \multicolumn{2}{|l|}{ Cys/Cys vs Ser/Ser } & \multicolumn{2}{|c|}{ Cys/Cys vs Ser/Ser+Ser/Cys } & \multicolumn{2}{|c|}{ Cys/Cys + Ser/Cys vs Ser/Ser } \\
\hline & & OR $(95 \% \mathrm{CI})$ & $I^{2}$ & OR $(95 \% \mathrm{CI})$ & $I^{2}$ & OR $(95 \% \mathrm{CI})$ & $I^{2}$ & OR $(95 \% \mathrm{CI})$ & $I^{2}$ \\
\hline Overall & 5 & $0.98(0.95-1.05)$ & 25.4 & $0.95(0.80-1.14)$ & 0.0 & $0.95(0.78-1.14)$ & 0.0 & $1.00(0.89-1.12)$ & 66.3 \\
\hline \multicolumn{10}{|l|}{ Ethnicity } \\
\hline Caucasians & 4 & $0.98(0.90-1.17)$ & 43.9 & $0.96(0.74-1.23)$ & 0.0 & $0.94(0.73-1.21)$ & 0.0 & $1.00(0.85-1.18)$ & 73.7 \\
\hline Asian & 1 & $0.98(0.87-1.09)$ & - & $0.95(0.75-1.21)$ & - & $0.96(0.73-1.26)$ & - & $0.98(0.89-1.08)$ & - \\
\hline \multicolumn{10}{|l|}{ Control population } \\
\hline Population-based & 3 & $0.93(0.79-1.11)$ & 54.1 & $0.95(0.73-1.33)$ & 28.7 & $0.98(0.72-1.34)$ & 0.0 & $0.96(0.74-1.26)$ & 81.9 \\
\hline Hospital-based & 2 & $1.01(0.92-1.10)$ & 0.0 & $0.94(0.76-1.16)$ & 0.0 & $0.93(0.73-1.17)$ & 0.0 & $1.03(0.95-1.11)$ & 3.0 \\
\hline \multicolumn{10}{|l|}{ Sample size } \\
\hline$>500$ subjects & 4 & $1.00(0.93-1.08)$ & 0.0 & $0.97(0.81-1.16)$ & 0.0 & $0.95(0.78-1.15)$ & 0.0 & $1.04(0.97-1.11)$ & 38.3 \\
\hline$\leq 500$ subjects & 1 & $0.73(0.55-0.97)$ & - & $0.80(0.49-1.32)$ & - & $0.97(0.48-1.95)$ & - & $0.69(0.52-0.92)$ & - \\
\hline \multicolumn{10}{|l|}{ Genotyping method } \\
\hline TaqMan & 3 & $1.02(0.94-1.10)$ & 0.0 & $1.01(0.83-1.22)$ & 0.0 & $0.98(0.79-1.21)$ & 0.0 & $1.08(0.95-1.19)$ & 56.1 \\
\hline Non-TaqMan & 2 & $0.86(0.66-1.12)$ & 64.4 & $0.80(0.54-1.20)$ & 0.0 & $0.85(0.56-1.28)$ & 0.0 & $0.84(0.60-1.20)$ & 79.6 \\
\hline
\end{tabular}

$N$ number of studies, $P$ the $P$ value used to test the heterogeneity, $C I$ confidence interval

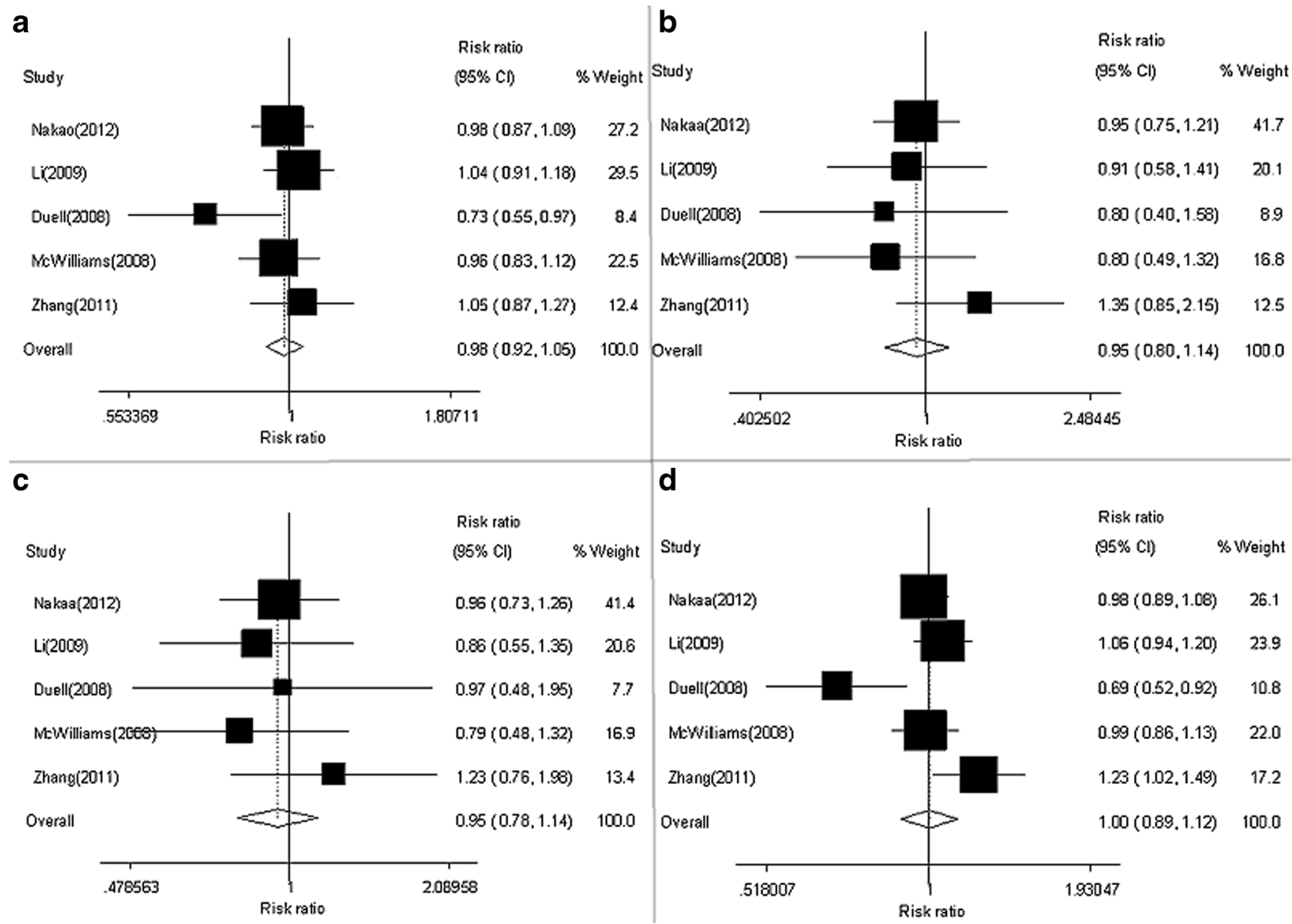

Fig. 1 Forest plots showed that OGG1 Ser326Cys polymorphism was not associated with PC susceptibility under all genetic models. a Allele contrast: Cys vs Ser; b homozygote model, Cys/Cys vs Ser/Ser; c

recessive model, Cys/Cys vs Ser/Ser+Ser/Cys; d dominant model, Cys/ Cys + Ser/Cys vs Ser/Ser 
Meta-analysis

As shown in Table 2, there is no significant association between the OGG1 Ser326Cys and PC risk was found in any of the genetic models. The results are as follow: Cys vs. Ser $(\mathrm{OR}=0.98,95 \% \mathrm{CI}=0.95-1.05$; Fig. 1a); Cys/Cys vs. Ser/Ser: OR=0.95, $95 \%$ CI=0.80-1.14 (Fig. 1b); Cys/Cys vs. Ser/Ser+Ser/Cys: OR=0.95, $95 \% \mathrm{CI}=0.78-1.14$ (Fig. 1c); $\mathrm{Cys} / \mathrm{Cys}+\mathrm{Ser} / \mathrm{Cys}$ vs. Ser/Ser $(\mathrm{OR}=1.00,95 \% \mathrm{CI}=0.89$ 1.12; Fig. 1d). Further subgroup analysis by ethnicity, source of controls, sample size, and genotyping method all yielded no statistically significant estimates (Table 2).

Test of heterogeneity

In the current study, significant heterogeneity was only found in dominant model (Cys/Cys + Ser/Cys vs Ser/Ser: $P=0.018$, $I^{2}=66.3 \%$ ). Then, we assessed the source of heterogeneity by ethnicity (Caucasians or Asian), source of control (population- or hospital-based), sample size ( $>500$ subjects or $\leq 500$ subjects), and genotyping method (TaqMan or non-TaqMan). As a result, the source of control $\left(\chi^{2}=12.23 ; d f=1 ; I^{2}=91.8 \%\right)$ and sample size $\left(\chi^{2}=2.10 ; d f=1 ; I^{2}=52.3 \%\right)$ were found contribute substantial heterogeneity.

Sensitivity analyses and publication bias

Sensitivity analysis was carried out to evaluate the influence of each individual study on the pooled OR. The results indicated that no individual study significantly impact on the overall results. The publication bias was assessed by Egger's test and funnel plot. No publication bias was detected for OGG1 Ser326Cys (the Egger's test result as follow: Cys vs. Ser: $P=0.256$, Cys/Cys vs. Ser/Ser: $P=0.877$, Cys/Cys vs. Ser/Ser+Ser/Cys: $P=0.982$, Cys/Cys + Ser/Cys vs. Ser/Ser $=$ 0.643). The shape of the funnel plot did not indicate any evidence of obvious asymmetry too (Fig. 2).
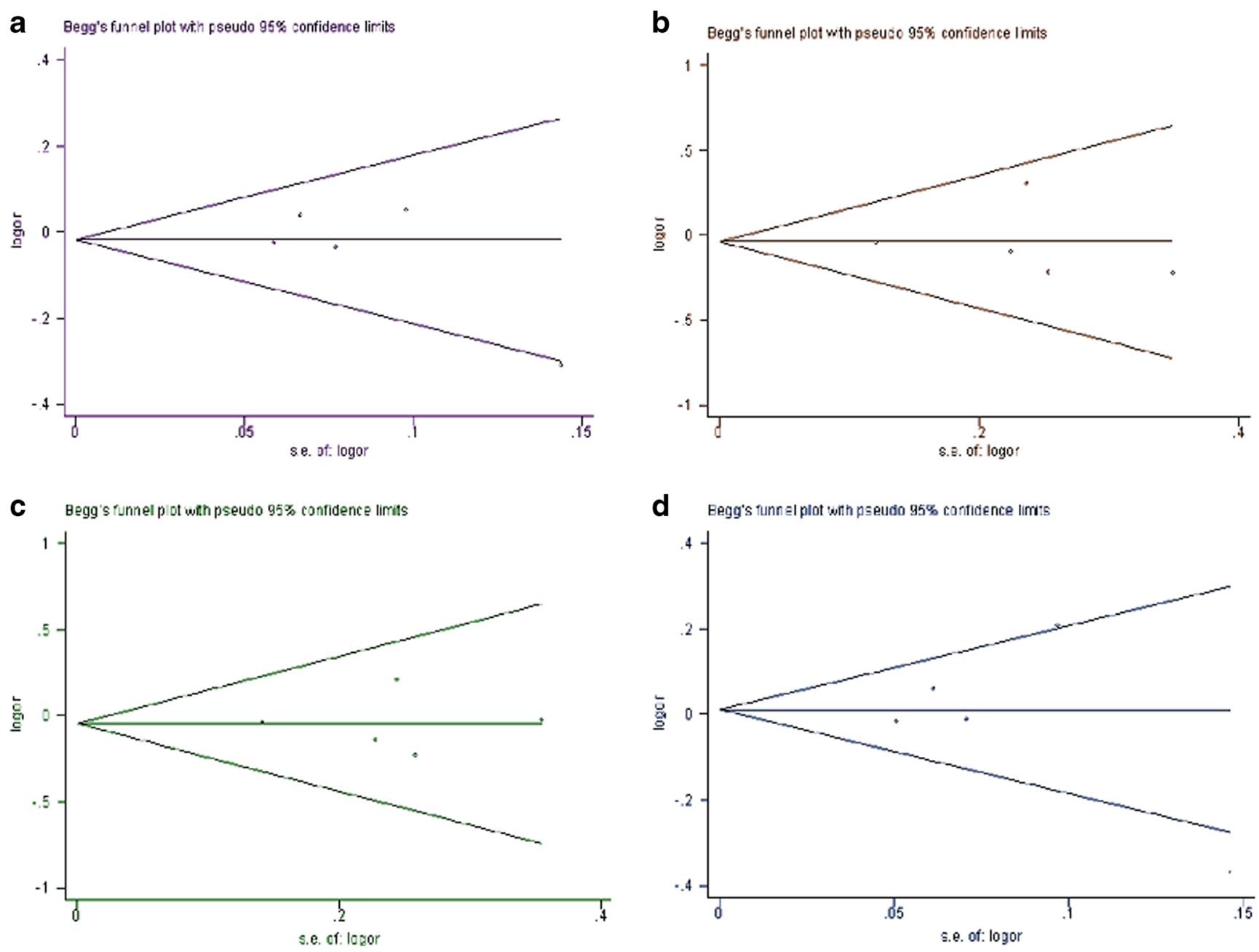

Fig. 2 Funnel plots did not indicate any evidence of obvious asymmetry under all genetic models. a Allele contrast, Cys vs Ser; b homozygote model: Cys/Cys vs Ser/Ser; $\mathbf{c}$ recessive model: Cys/Cys vs Ser/Ser+Ser/Cys; $\mathbf{d}$ dominant model: Cys/Cys+Ser/Cys vs Ser/Ser 


\section{Discussion}

In recent years, a number of studies have carried out to explore the $O G G 1$ Ser326Cys polymorphism with cancers risk including lung cancer, breast cancer, colorectal cancer, and PC. Unfortunately, previous findings of OGG1 Ser326Cys polymorphism on cancer susceptibility were controversial or ambiguous. Therefore, some meta-analyses were performed to solve the phenomenon. Zhang et al. reported that the $O G G 1$ Ser326Cys polymorphism is not associated with CRC risk and Wang et al. concluded that the OGG1 Ser326Cys polymorphism might not be a potential candidate risk factor for the development of gastric cancer [33, 34]. However, Yuan et al. suggested that the $O G G 1326 \mathrm{Cys}$ allele plays a significant protective effect to breast cancer in European women and Duan et al. established solid statistical evidence for an association between the $O G G 1 \mathrm{Cys} / \mathrm{Cys}$ genotype and lung cancer risk $[35,36]$. This phenomenon indicates that the OGG1 Ser326Cys polymorphism exerts different effect on various types of cancers. So, it is necessary for us to get a better understanding of $O G G 1$ Ser326Cys polymorphism on PC susceptibility, especially when inclusive and controversial findings still exist. In our present meta-analysis, $O G G 1$ Ser326Cys polymorphism was not significantly associated with PC risk. Subgroup analysis was based on ethnicity, source of control, sample size, and genotyping method; we could not achieve a significant association between $O G G 1$ Ser326Cys polymorphism and PC susceptibility.

When performing meta-analysis, testing the heterogeneity among studies is very important. In the current study, significant heterogeneity was only found in the dominant model. Then, we did subgroup analysis to search the source of heterogeneity. Interestingly, it seems that the ethnicity is not the source of heterogeneity, suggesting that $O G G 1$ Ser326Cys polymorphism may not have race-specific effects on PC susceptibility. We found that the source of control and sample size may contribute substantial heterogeneity. It is possible that some limitations of recruited studies may partially contribute to the observed heterogeneity. For this reason, we conducted analyses using the random effects model. In addition, publication bias is another aspect which may make a negative effect on our meta-analysis. Both funnel plot and Egger's test were applied to test the publication bias. Our results suggest that the publication bias have little effect on the results of our study, and the results of our meta-analysis are relatively stable.

Although comprehensive analysis was conducted to explore the association between $O G G 1$ Ser326Cys polymorphism and PC susceptibility, there are still some limitations. Firstly, the primary studies mainly provided data towards Caucasians; therefore, other ethnicities should be researched in future studies. Secondly, only three studies used controls that were populationbased. Other articles used hospital-based controls, which may not be representative of the general population. Thirdly, the number of samples included in the meta-analysis was relatively small.

In spite of the shortages above, our meta-analysis also had several advantages. Firstly, strict searching strategy which combines computer-assisted with manual search makes the eligible studies included as much as possible. Secondly, the quality of case-control studies met our inclusion criteria and was satisfactory, and the sensitivity analysis and publication bias analysis indicated the stability and credibility of the metaanalysis, which leads to a more convincing result. More importantly, the process of literature selection, data extraction, and data analysis were well designed and conducted.

In conclusion, this is the first meta-analysis evaluating the association between $O G G 1$ Ser326Cys polymorphism and $\mathrm{PC}$ susceptibility. The pooled results suggest that the $O G G 1$ Ser326Cys polymorphism may not be associated with PC susceptibility. Considering the limited sample size and ethnicities included in the meta-analysis, further larger-scaled and well-designed studies are needed to confirm our results.

\section{Conflicts of interest None.}

Open Access This article is distributed under the terms of the Creative Commons Attribution License which permits any use, distribution, and reproduction in any medium, provided the original author(s) and the source are credited.

\section{References}

1. Jemal A, Siegel R, Ward E, Hao Y, Xu J, Murray T, et al. Cancer statistics, 2008. CA Cancer J Clin. 2008;58:71-96.

2. Jemal A, Bray F, Center MM, Ferlay J, Ward E, Forman D. Global cancer statistics. CA Cancer J Clin. 2011;61:69-90.

3. Ghaneh P, Costello E, Neoptolemos JP. Biology and management of pancreatic cancer. Gut. 2007;56:1134-52.

4. Siegel R, Naishadham D, Jemal A. Cancer statistics, 2012. CA Cancer J Clin. 2012;62:10-29.

5. Hariharan D, Saied A, Kocher HM. Analysis of mortality rates for pancreatic cancer across the world. HPB: Off J Int Hepato Pancreato Biliary Assoc. 2008;10:58-62.

6. Le Scodan R, Mornex F, Girard N, Mercier C, Valette PJ, Ychou M, et al. Preoperative chemoradiation in potentially resectable pancreatic adenocarcinoma: feasibility, treatment effect evaluation and prognostic factors, analysis of the SFRO-FFCD 9704 trial and literature review. Ann Oncol: Off J Eur Soc Med Oncol/ESMO. 2009;20:1387-96.

7. Bednar F, Simeone DM. Pancreatic cancer stem cell biology and its therapeutic implications. J Gastroenterol. 2011;46:1345-52.

8. Gutierrez ML, Munoz-Bellvis L, Abad Mdel M, Bengoechea O, Gonzalez-Gonzalez M, Orfao A, et al. Association between genetic subgroups of pancreatic ductal adenocarcinoma defined by high density 500 k SNP-arrays and tumor histopathology. PLoS One. 2011;6:e22315.

9. Rizzato C, Campa D, Giese N, Werner J, Rachakonda PS, Kumar R, et al. Pancreatic cancer susceptibility loci and their role in survival. PLoS One. 2011;6:e27921.

10. Lucenteforte E, La Vecchia C, Silverman D, Petersen GM, Bracci PM, Ji BT, et al. Alcohol consumption and pancreatic cancer: a pooled analysis in the International Pancreatic Cancer Case-Control 
Consortium (PANC4). Ann Oncol: Off J Eur Soc Med Oncol / ESMO. 2012;23:374-82.

11. Iodice S, Gandini S, Maisonneuve P, Lowenfels AB. Tobacco and the risk of pancreatic cancer: a review and meta-analysis. Langenbeck's Arch Surg/Deut Ges Chir. 2008;393:535-45.

12. Aune D, Chan DS, Vieira AR, Navarro Rosenblatt DA, Vieira R, Greenwood DC, et al. Dietary fructose, carbohydrates, glycemic indices and pancreatic cancer risk: a systematic review and metaanalysis of cohort studies. Ann Oncol: Off J Eur Soc Med Oncol / ESMO. 2012;23:2536-46.

13. Li G, Chen Q, Wang L, Ke D, Yuan Z. Association between FTO gene polymorphism and cancer risk: evidence from 16,277 cases and 31,153 controls. Tumour Biol: J Int Soc Oncodev Biol Med. 2012;33:1237-43.

14. Dong LM, Potter JD, White E, Ulrich CM, Cardon LR, Peters U. Genetic susceptibility to cancer: the role of polymorphisms in candidate genes. JAMA: J Am Med Assoc. 2008;299:2423-36.

15. Yun SJ, Ha YS, Chae Y, Kim JS, Kim IY, Kim WJ. The hOGG1 mutant genotype is associated with prostate cancer susceptibility and aggressive clinicopathological characteristics in the Korean population. Ann Oncol: Off J Eur Soc Med Oncol / ESMO. 2012;23:401-5.

16. Yang N, Chaudhry MA, Wallace SS. Base excision repair by hNTH1 and hOGG1: a two edged sword in the processing of DNA damage in gamma-irradiated human cells. DNA Repair. 2006;5:43-51.

17. Dherin C, Radicella JP, Dizdaroglu M, Boiteux S. Excision of oxidatively damaged DNA bases by the human alpha-hOGG1 protein and the polymorphic alpha-hOGG1(ser326cys) protein which is frequently found in human populations. Nucleic Acids Res. 1999;27:4001-7.

18. Gerhard DS, Wagner L, Feingold EA, Shenmen CM, Grouse LH, Schuler G, et al. The status, quality, and expansion of the NIH fulllength cDNA project: the Mammalian Gene Collection (MGC). Genome Res. 2004;14:2121-7.

19. Vodicka P, Stetina R, Polakova V, Tulupova E, Naccarati A, Vodickova L, et al. Association of DNA repair polymorphisms with DNA repair functional outcomes in healthy human subjects. Carcinogenesis. 2007;28:657-64.

20. Weiss JM, Goode EL, Ladiges WC, Ulrich CM. Polymorphic variation in hOGG1 and risk of cancer: a review of the functional and epidemiologic literature. Mol Carcinog. 2005;42:127-41.

21. Kohno T, Shinmura K, Tosaka M, Tani M, Kim SR, Sugimura H, et al. Genetic polymorphisms and alternative splicing of the hOGG1 gene, that is involved in the repair of 8-hydroxyguanine in damaged DNA. Oncogene. 1998;16:3219-25.

22. Nakao M, Hosono S, Ito H, Watanabe M, Mizuno N, Sato S, et al. Selected polymorphisms of base excision repair genes and pancreatic cancer risk in Japanese. J Epidemiol/Jpn Epidemiol Assoc. 2012;22: 477-83.

23. Li D, Suzuki H, Liu B, Morris J, Liu J, Okazaki T, et al. DNA repair gene polymorphisms and risk of pancreatic cancer. Clin Cancer Res: Off J Am Assoc Cancer Res. 2009;15:740-6.

24. Duell EJ, Bracci PM, Moore JH, Burk RD, Kelsey KT, Holly EA. Detecting pathway-based gene-gene and gene-environment interactions in pancreatic cancer. Cancer Epidemiol Biomarkers Prev: Publ Am Assoc Cancer Res CoCosponsored Am Soc Prev Oncol. 2008;17:1470-9.

25. McWilliams RR, Bamlet WR, Cunningham JM, Goode EL, de Andrade M, Boardman LA, et al. Polymorphisms in DNA repair genes, smoking, and pancreatic adenocarcinoma risk. Cancer Res. 2008;68:4928-35.

26. Zhang J, Zhang X, Dhakal IB, Gross MD, Kadlubar FF, Anderson KE. Sequence variants in antioxidant defense and DNA repair genes, dietary antioxidants, and pancreatic cancer risk. Int J Mol Epidemiol Genet. 2011;2:236-44.

27. Cochran WG. The comparison of percentages in matched samples. Biometrika. 1950;37:256-66.

28. Mantel N, Haenszel W. Statistical aspects of the analysis of data from retrospective studies of disease. J Natl Cancer Inst. 1959;22:719-48.

29. DerSimonian R, Laird N. Meta-analysis in clinical trials. Control Clin Trials. 1986;7:177-88.

30. Egger M, Davey Smith G, Schneider M, Minder C. Bias in metaanalysis detected by a simple, graphical test. BMJ. 1997;315:629-34.

31. Stuck AE, Rubenstein LZ, Wieland D. Bias in meta-analysis detected by a simple, graphical test. Asymmetry detected in funnel plot was probably due to true heterogeneity. BMJ. 1998;316:469. author reply $470-461$.

32. Tobias A, Campbell MJ. Modelling influenza epidemics in the relation between black smoke and total mortality. A sensitivity analysis. J Epidemiol Community Health. 1999;53:583-4.

33. Yuan W, Xu L, Feng Y, Yang Y, Chen W, Wang J, et al. The hOGG1 ser326cys polymorphism and breast cancer risk: a meta-analysis. Breast Cancer Res Treat. 2010;122:835-42.

34. Zhang Y, He BS, Pan YQ, Xu YQ, Wang SK. Association of OGG1 Ser326cys polymorphism with colorectal cancer risk: a metaanalysis. Int J Color Dis. 2011;26:1525-30.

35. Wang Z, Hu J, Cai W, Zhong J. Lack of association between the 8oxoguanine DNA glycosylase gene ser326cys polymorphism and gastric cancer: evidence from a meta-analysis. Asian Pac J Cancer Prev: APJCP. 2011;12:3427-31.

36. Duan WX, Hua RX, Yi W, Shen LJ, Jin ZX, Zhao YH, et al. The association between ogg1 ser326cys polymorphism and lung cancer susceptibility: a meta-analysis of 27 studies. PLoS One. 2012;7:e35970. 